\title{
Urban Area Detection Using Local Feature Points and Spatial Voting
}

\author{
Beril Sırmaçek, Student Member, IEEE, and Cem Ünsalan, Member, IEEE
}

\begin{abstract}
Automatically detecting and monitoring urban regions is an important problem in remote sensing. Very high resolution aerial and satellite images provide valuable information to solve this problem. However, they are not sufficient alone for two main reasons. First, a human expert should analyze these very large images. There may be some errors in the operation. Second, the urban area is dynamic. Therefore, detection should be done periodically, and this is time consuming. To handle these shortcomings, an automated system is needed to detect the urban area from aerial and satellite images. In this letter, we propose such a method based on local feature point extraction using Gabor filters. We use these local feature points to vote for the candidate urban areas. Then, we detect the urban area using an optimal decision-making approach on the vote distribution. We test our method on a diverse panchromatic aerial and Ikonos satellite image set. Our test results indicate the possible use of our method in practical applications.
\end{abstract}

Index Terms-Aerial images, Gabor filter, local feature points, satellite images, spatial voting, urban area detection.

\section{INTRODUCTION}

$\mathbf{M}$ ONITORING urbanization may help government agencies and urban region planners in updating land maps and forming long-term plans accordingly. Very high resolution aerial and satellite images provide valuable information for this purpose. Unfortunately, these images cover very large areas. Therefore, their manual inspection is very hard and prone to errors. Furthermore, urban areas are dynamic environments. Hence, they should be monitored periodically. Because of these difficulties, manually monitoring urbanization using very high resolution aerial and satellite images is not feasible. Therefore, in this letter, we propose an automated system for the first step of urban monitoring, i.e., detecting urban areas.

In the literature, many researchers considered the urban area detection problem using automated techniques. Karathanassi et al. [6] used building density information to classify residential regions. They benefit from texture information and segmentation to extract the residential areas. Unfortunately, they had several parameters to be adjusted manually. Benediktsson et al. [1] used mathematical morphological operations to extract structural information to detect the urban area in satellite images. They benefit from neural networks for

Manuscript received June 10, 2009; revised July 10, 2009. First published September 11, 2009; current version published January 13, 2010. This work was supported by Ístanbul Büyükçehir Belediyesi.

The authors are with the Computer Vision Research Laboratory, Department of Electrical and Electronics Engineering, Yeditepe University, 34755 Istanbul, Turkey (e-mail: bsirmacek@yeditepe.edu.tr; unsalan@yeditepe.edu.tr).

Color versions of one or more of the figures in this paper are available online at http://ieeexplore.iee.org.

Digital Object Identifier 10.1109/LGRS.2009.2028744 classification purposes. Therefore, they need training data to detect the urban areas. Ünsalan and Boyer [12]-[14] used structural features to classify urban regions in panchromatic satellite images. Since they use statistical classifiers, they also need training data to detect the urban area in the image. In a following study, Ünsalan and Boyer [15] associated structural features with graph theoretical measures in order to grade the satellite images and extract the residential regions from them. In a related study, Sırmaçek and Ünsalan [10] used scale-invariant feature transform (SIFT) and graph theory to detect urban areas and buildings in grayscale Ikonos images. They used template building images for this purpose. Although graph theoretical methods are suitable for urban area detection, they need considerable computation power and operation time. Fonte et al. [5] considered corner detectors to obtain the type of structure in a satellite image. They concluded that corner detectors might give distinctive information on the type of structure in an image. Bhagavathy and Manjunath [2] used texture motifs for modeling and detecting regions (such as golf parks and harbors) in satellite images. They focused on repetitive patterns in the image. Bruzzone and Carlin [3] proposed a context-based system to classify very high resolution satellite images. They used support vector machines fed with a novel feature extractor. Fauvel et al. [4] fused different classifiers to extract and classify urban regions in panchromatic satellite images. Zhong and Wang [17] extracted urban regions in grayscale satellite images using a multiple-classifier approach. These last three studies also need training data for urban area classification.

In this letter, we propose a novel method to detect urban areas in very high resolution panchromatic aerial and satellite images. Different from most of the studies in the literature, our method does not need any training data for urban area detection. Instead, we first use Gabor filters to extract spatial building characteristics (such as edges and corners) in different orientations. Then, we process the filter outputs to obtain local feature points in the image. We form a voting matrix using them. Finally, we label the urban area in a given image by an optimal adaptive decision-making approach. Our method is able to detect the urban areas as long as buildings are dense. Moreover, we do not have any other constraints. To provide experimental justification to our method, we tested it on diverse aerial and Ikonos satellite images. We obtained encouraging results.

\section{Local Feature Point Extraction}

We detect the urban area in a given test image using local feature points. The first step to extract them from an image is smoothing it by median filtering [11]. This step eliminates 
small noise terms in the image. Then, we apply Gabor filtering in different directions. The maxima in these filter responses lead to local feature points. Next, we will explore these steps in detail.

\section{A. Gabor Filtering}

Gabor filters are extensively used in texture segmentation and object recognition [7]. They exhibit desirable characteristics as spatial locality and orientation selectivity [16]. Mathematically, the 2-D Gabor filter can be defined as the product of a Gaussian and a complex exponential function as

$$
F_{\varphi}(x, y)=\frac{1}{2 \pi \sigma_{g}^{2}} \exp \left(-\frac{u^{2}+v^{2}}{2 \sigma_{g}^{2}}\right) \exp (j 2 \pi f u)
$$

where $u=x \cos \varphi+y \sin \varphi$ and $v=-x \sin \varphi+y \cos \varphi$. $f$ is the frequency of the complex exponential signal, $\varphi$ is the direction of the Gabor filter, and $\sigma_{g}$ is the scale parameter. These parameters should be adjusted with respect to the image resolution at hand. In this letter, we explore the effect of these parameters in Section IV-A.

We can detect the edge-oriented urban characteristics (such as building edges) in a test image using Gabor filtering. Therefore, for a test image $I(x, y)$ (with size $N \times M$ ), we benefit from the real part of the Gabor filter response as

$$
G_{\varphi}(x, y)=\mathfrak{R e}\left\{I(x, y) * F_{\varphi}(x, y)\right\}
$$

where * stands for the 2-D convolution operation. $G_{\varphi}(x, y)$ is the maximum for image regions having similar characteristics with the filter. In the next section, we use this information to extract the local feature points.

\section{B. Local Feature Points}

To extract the local feature points, we first search for the local maxima in $G_{\varphi}(x, y)$ for $x=1, \ldots, N$ and $y=1, \ldots, M$. If any pixel $\left(x_{o}, y_{o}\right)$ in $G_{\varphi}(x, y)$ has the largest value among its neighbors, $G_{\varphi}\left(x_{o}, y_{o}\right)>G_{\varphi}\left(x_{n}, y_{n}\right) \forall\left(x_{n}, y_{n}\right) \in\left\{\left(x_{o}-\right.\right.$ $\left.\left.1, y_{o}-1\right),\left(x_{o}, y_{o}-1\right), \ldots,\left(x_{o}+1, y_{o}+1\right)\right\}$; we call it as a local maximum. It is a candidate for being a local feature point. Next, we check the amplitude of the filter response $G_{\varphi}\left(x_{o}, y_{o}\right)$. We call our local maximum $\left(x_{o}, y_{o}\right)$ as a candidate local feature point if and only if $G_{\varphi}\left(x_{o}, y_{o}\right)>\alpha$. To handle different images, we obtain $\alpha$ using Otsu's method on $G_{\varphi}(x, y)$ in an adaptive manner for each image separately [8]. Therefore, we eliminate the weak candidate local feature points in future calculations.

To represent each candidate local feature point further, we assign a weight $w_{o}$ to it as follows. We first threshold $G_{\varphi}(x, y)$ with $\alpha$ and obtain a binary image $B_{\varphi}(x, y)$. In this image, pixels having value one correspond to strong responses. We obtain connected pixels to $\left(x_{o}, y_{o}\right)$ in $B_{\varphi}\left(x_{o}, y_{o}\right)$. By definition, two pixels are connected (in a binary image) to each other if there is a path (of pixels with value one) connecting them [11]. As we obtain all the connected pixels to $\left(x_{o}, y_{o}\right)$, we assign their sum as the weight $w_{o}$. Therefore, if a candidate local feature point has more connected pixels, it has more weight. We expect the candidate local feature points to represent the urban character-
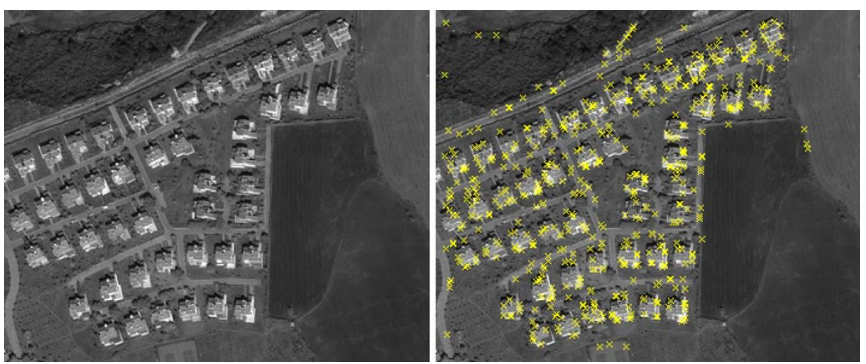

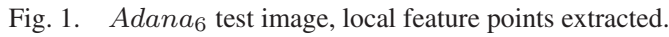

istics such as building clusters. Unfortunately, all the candidate local feature points may not represent reliable information on the urban area. Therefore, we discard the candidate local feature points having weight $w_{o}$ that is less than 20 pixels. Although we applied the same weight threshold value for both aerial and satellite images (having different characteristics) in this letter, the reader may need to adjust it with respect to the test image at hand. Finally, we obtain the local feature points for the given $\varphi$ direction.

We apply this procedure in all $\varphi$ directions and obtain a total of $K$ local feature points as $\left(x_{k}, y_{k}\right)$ with their weights $w_{k}$ for $k=1, \ldots, K$. We expect these local feature points to be located on the building edges in the image. We provide such an example on the sample Adana ${ }_{6}$ satellite test image in Fig. 1. As can be seen, most local feature points are located on the building edges in this image.

In the literature, there are also more complex feature point extraction methods [9]. However, for urban area detection, we do not need perfect local point extraction from the image. Since we use their ensemble, missing a few local feature points does not affect the performance of our urban area detection method. We will explore urban area detection using local feature points next.

\section{URban AREA Detection}

As we obtain the local feature points, the next step is detecting the urban area using them. Therefore, we form a voting matrix based on spatial voting first. Then, we apply an optimum decision-making method to detect the urban area from the voting matrix. We will explain these steps in detail next.

\section{A. Voting Matrix Formation}

To detect an urban area, we should have many local feature points in it. These should also be closely located in the spatial domain. Therefore, we define a voting matrix based on the extracted local feature points as follows. We assume that, around each local feature point, there is a high possibility of an urban characteristic (such as a building). Therefore, each local feature point has the highest vote at its spatial coordinate $\left(x_{k}, y_{k}\right)$, and its votes decrease with respect to the spatial distance. Based on this definition, we form the voting matrix for $x=1, \ldots, N$ and $y=1, \ldots, M$ as

$$
V(x, y)=\sum_{k=1}^{K} \frac{1}{2 \pi \sigma_{k}^{2}} \exp \left(-\frac{\left(x-x_{k}\right)^{2}+\left(y-y_{k}\right)^{2}}{2 \sigma_{k}^{2}}\right)
$$




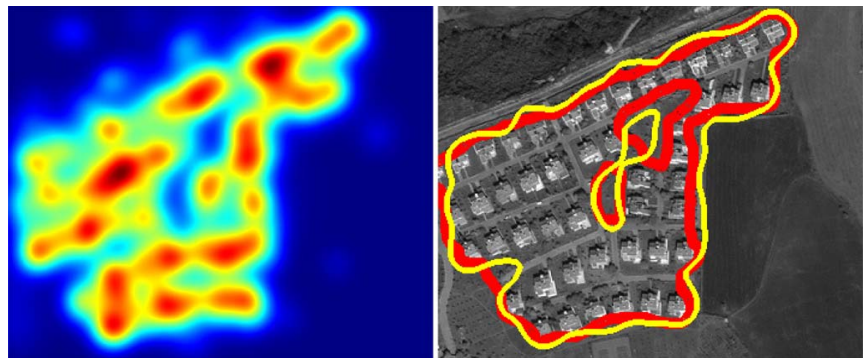

Fig. 2. Adana A $_{6}$ test image, the voting matrix obtained and the urban area detected (with ground truth data).

where $\sigma_{k}$ is the parameter for voting proximity for each local feature point. For our test images, we pick $\sigma_{k}=5 \times w_{k}$ to add some tolerance for voting. If $\sigma_{k}$ has higher values, each local feature point will have a wider spatial effect on the voting matrix. Therefore, false alarms in urban area detection will increase. On the other hand, if $\sigma_{k}$ has lower values, each local feature point will have a narrower spatial effect. Hence, the correct urban area detection results will decrease.

For the Adana $_{6}$ test image, we provide the voting matrix in Fig. 2. In this figure, the vote values are color coded (red corresponds to the highest vote value and blue to the lowest vote value). As can be seen, the votes are cumulated around the buildings.

\section{B. Optimum Decision Making}

Locations with high votes in $V(x, y)$ are possible urban area pixels, and locations with low votes are possible nonurban area pixels in the image. Therefore, we expect to have a bimodal pixel distribution on $V(x, y)$ (one peak corresponds to the urban area and the other to the nonurban area pixel votes). We use this information and Otsu's method to detect the urban area [8]. Based on Bayes decision criteria, Otsu's method finds the optimal threshold level (assuming Gaussian probability density functions) between the urban and nonurban pixel votes. In this letter, the threshold value is adaptively obtained for each test image separately. Since this operation is adaptive, we do not need any manual (or predefined) parameter adjustments.

For the $A_{\text {dana }}$ satellite test image, we provide the voting matrix pixel distribution in Fig. 3. As can be seen in this distribution, there are two peaks (one corresponding to urban and the other to the nonurban area votes). In the same figure, we also provide the optimum decision boundary obtained by Otsu's method by a red dashed vertical line. As can be seen, the decision boundary is correctly located. Based on this threshold value, we provide the detected urban area (as a yellow curve) from the Adana $_{6}$ image in Fig. 2. In the same figure, we also provide our ground truth data for the $A_{d a n a_{6}}$ test image as a red thick curve. As can be seen, our detection result closely fits to the ground truth data.

Aside from thresholding with Otsu's method, we also consider the overall votes in the test image. If their accumulation is not sufficient, we assume that they cannot represent an urban area. To perform this test on an image basis, we assume that, if the sum of the votes does not exceed $5 \%$ of the image size, there is no urban area there. After extensive testing, we obtained this

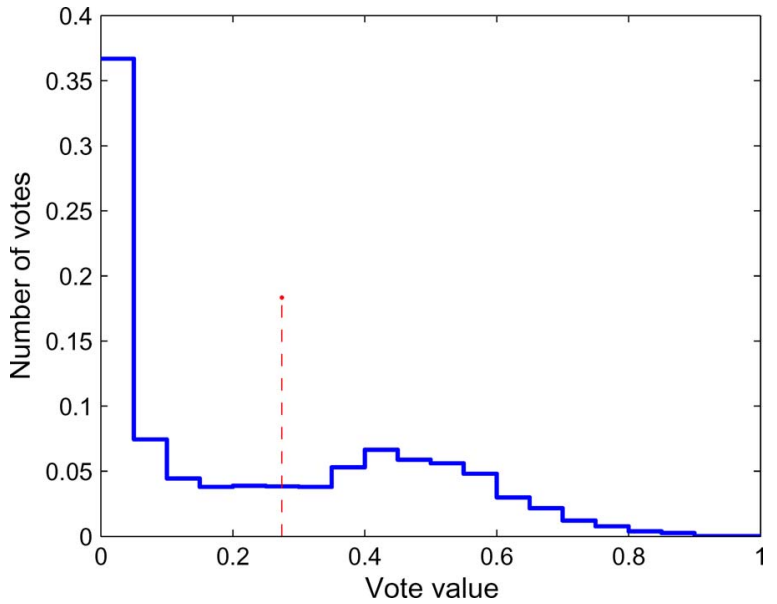

Fig. 3. Voting matrix pixel distribution and the optimum threshold value for the Adana $_{6}$ test image.

value for our test images. However, it is not strict and can be changed in a relaxed manner.

\section{EXPERIMENTS}

To test the performance of our urban area detection method, we use 21 aerial and 31 panchromatic Ikonos satellite images. Our aerial images have $0.3-\mathrm{m}$ spatial resolution, and the Ikonos satellite images have 1-m spatial resolution. These aerial and satellite images naturally have different acquisition sensor characteristics. Therefore, the experimental test results (provided in this section) can be taken as independent of the acquisition sensor characteristics and spatial resolution. Our test images are also specifically selected to represent the wide and diverse urban area characteristics. On the Ikonos images, we first provide the experimental justification for the selected parameter values. In the following two sections, we provide the overall urban area detection performance of our method on the satellite and aerial images separately. We quantify these tests by reporting the urban area detection percentage $P_{d}$ (the ratio of the correctly detected urban area pixels to the manually labeled urban area pixels) and the overall false alarm percentage $P_{f}$ (the ratio of the false detected pixels to the manually labeled urban area pixels). In forming the ground truth (manually labeled urban area), we label a region as urban if it contains building clusters, gardens around them, and nearby street segments joining them as we did in our previous study [10]. We also provide the computation times for each operation in our urban area detection method on a sample test image. Finally, we compare our method with our previously published urban area detection method [10]. There, we used SIFT and graph theoretical tools for urban area detection.

\section{A. Tests on Parameter Values}

Although we do not have many parameters in our urban area detection method, we provide their effect on the final detection results for the satellite images in this section. We first comment on the Gabor filter parameters. We observed that, in the panchromatic Ikonos images, building edges can be represented 
TABLE I

EFFEct of Gabor Filtering Directions on Urban Area Detection

\begin{tabular}{|c|r|r|}
\hline Number of $\varphi$ & $\mathbf{P}_{\mathbf{d}}(\%)$ & $\mathbf{P}_{\mathbf{f}}(\%)$ \\
\hline 4 & 88.39 & 11.55 \\
\hline 6 & 88.63 & 8.21 \\
\hline 8 & 88.47 & 6.30 \\
\hline $\mathbf{1 0}$ & $\mathbf{8 9 . 3 3}$ & $\mathbf{5 . 9 1}$ \\
\hline 12 & 88.79 & 5.91 \\
\hline 14 & 90.79 & 5.99 \\
\hline
\end{tabular}

TABLE II

EfFect of Median Filtering on Urban Area Detection

\begin{tabular}{|c|r|r|}
\hline Median filter size & $\mathbf{P}_{\mathbf{d}}(\%)$ & $\mathbf{P}_{\mathbf{f}}(\%)$ \\
\hline No filtering & 95.04 & 31.76 \\
\hline $3 \times 3$ & 94.13 & 16.75 \\
\hline $\mathbf{5} \times \mathbf{5}$ & $\mathbf{8 9 . 3 3}$ & $\mathbf{5 . 9 1}$ \\
\hline $7 \times 7$ & 84.29 & 5.15 \\
\hline
\end{tabular}

as a ramp edge with three- to four-pixel width. Therefore, in this letter, we picked $\sigma_{g}=1.5$ and $f=0.65$ after extensive testing. We also used the same parameter values for the aerial test images without any problem. These two parameters should be adjusted with respect to the building characteristics in the test image. To cover the differently oriented building edges, we tested different $\varphi$ values. We provide different settings in Table I. In these tests, we use 31 Ikonos images to eliminate image dependence.

As can be seen in Table I, the number of Gabor filtering directions has effect on decreasing $P_{f}$. Based on this test, we can conclude that choosing ten different directions for Gabor filtering $(\varphi=\{0, \pi / 10,2 \pi / 10, \ldots, 9 \pi / 10\} \mathrm{rad})$ is suitable for urban area detection. To note here, choosing 6,8 , or 12 directions also provides similar $P_{d}$ and $P_{f}$ values. Therefore, we can conclude that our method does not specifically depend on the total Gabor filtering direction.

We then test the effect of median filtering on the final detection result. We provide tests with different median filter sizes in Table II. Again, in these tests, we use 31 Ikonos images to eliminate image dependence.

As can be seen in Table II, without median filtering, $P_{f}$ is fairly high. Therefore, we need a median filtering operation. As can be seen, a $5 \times 5$ median filtering gives reasonable $P_{d}$ and $P_{f}$ values.

For the aerial images, we also use the same settings. However, to make the resolution of the satellite and aerial images similar, we downsampled the aerial images by 0.5 . Next, we will discuss the overall urban area detection performance of our method on the aerial and satellite images.

\section{B. Overall Performance on Satellite Images}

The overall performance of our method on the 31 satellite images having 775714 urban area pixels is $P_{d}=89.33 \%$ and $P_{f}=5.91 \%$. Therefore, our method was able to detect 692926 urban area pixels correctly with 45854 false alarm pixels. This is a fairly good urban area detection result on such a diverse satellite image set. We also provide a sample test image for the urban area detection results for the satellite images in Fig. 4. As can be seen in this figure, all the urban regions with different building characteristics are correctly detected.

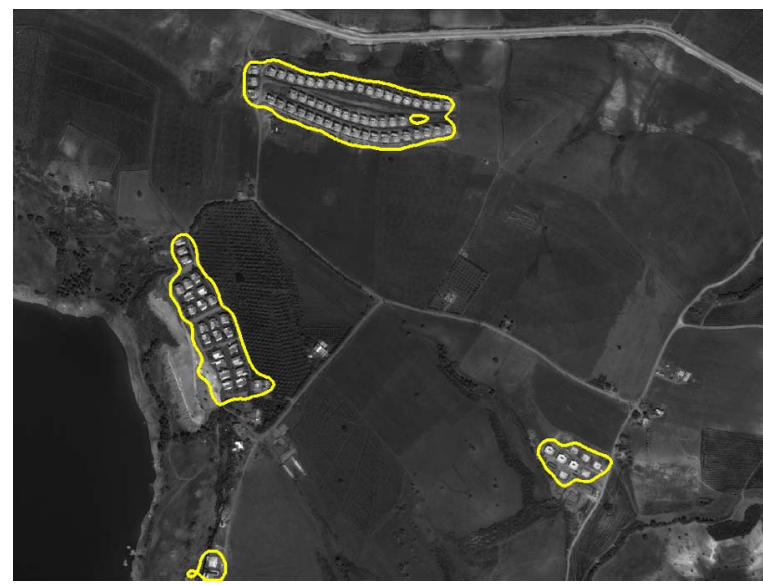

Fig. 4. Urban area detection result on a sample satellite image.

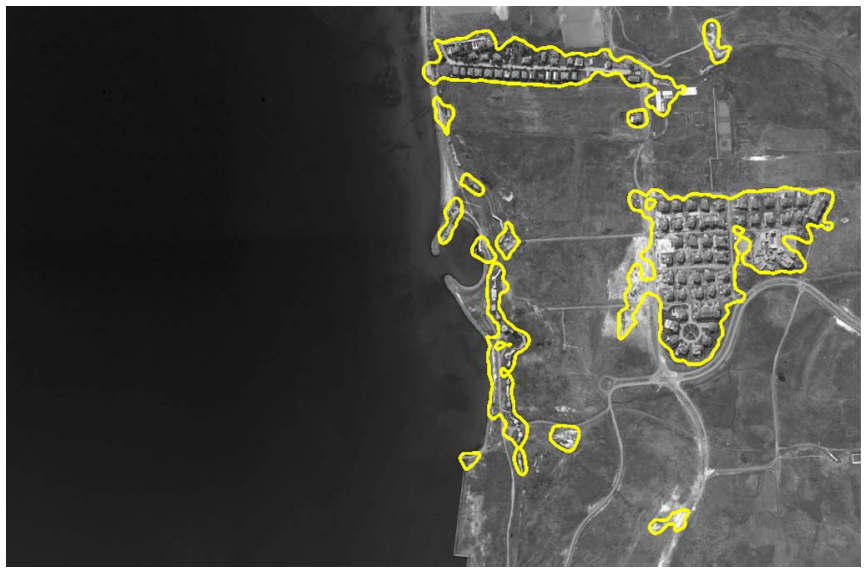

Fig. 5. Urban area detection result on a sample aerial image.

\section{Overall Performance on Aerial Images}

Next, we will discuss the overall performance of our method on the 21 aerial images. In this test, we have 1688936 urban area pixels. We obtained $P_{d}=85.93 \%$ with $P_{f}=10.95 \%$ for urban area detection. Therefore, 1451225 urban area pixels are correctly detected, with 184851 false alarm pixels. Similar to the satellite images test, this result on such a data set is fairly good. We also provide a sample test image for the urban area detection results for the aerial images in Fig. 5. As can be seen in this figure, all the urban regions with different building characteristics are correctly detected.

\section{Computation Time}

The other important property of our method is its computation time. To provide an idea about the computation time needed for our method, we next consider the CPU timings for each step on the $A_{d a n a_{8}}$ satellite test image (having a size of $235 \times 265$ pixels). We picked this image as a benchmark in our previous study [10]. Therefore, we pick the same image in this letter to compare both methods. In reporting the computation times, we used a PC with Intel Core2Duo processor with 2.13-GHz clock speed and 4 GB of RAM. We used MATLAB as our coding platform. After testing, we obtained that, for the $A d a n a_{8}$ image, Gabor filtering needs $0.61 \mathrm{~s}$, local feature point 
extraction needs $0.94 \mathrm{~s}$, and spatial voting needs $2.30 \mathrm{~s}$. The total time needed for urban area detection is $3.85 \mathrm{~s}$. This is a fairly good timing to obtain the urban area.

\section{E. Comparison With SIFT-Based Method}

Finally, we compare our new urban area detection method with that in our previous study based on SIFT and graph theory [10]. In this section, we use the same data set in our previous study (without the final two Adana satellite images and all the aerial images we used in this study). The overall urban area detection performance there was $P_{d}=89.62 \%$ with $P_{f}=8.03 \%$. On the same data set, with our new method, we obtain $P_{d}=89.09 \%$ with $P_{f}=5.88 \%$. The detection and false alarm rates are almost the same. Interested readers can visually compare the detection results on the $A d_{a n a} a_{6}$ satellite test image both with the present method and the SIFT-based method by checking [10].

To compare both methods in terms of CPU timings, we pick the Adana $_{8}$ satellite test image as a benchmark. The time needed for the SIFT-based method was $81.97 \mathrm{~s}$. With our new method, we only need $3.85 \mathrm{~s}$. Therefore, our new method is fairly fast and almost has the same performance with our SIFTbased urban area detection method. Moreover, we do not need any template building images (as in the SIFT-based method) to detect the urban areas in this new method. To note here, in our SIFT-based method, we also detect the buildings after detecting the urban area.

\section{CONCLUSION}

This letter focuses on urban area detection using very high resolution aerial and satellite images. It may be taken as the first step in monitoring urbanization. Our method depends on local feature point extraction using Gabor filtering. We use these local feature points in forming a spatial voting matrix. Then, by using an optimum decision-making approach, we are able to detect the urban area in a given aerial or satellite image. After extensive testings, we obtained very encouraging results with our method. Comparing with an existing algorithm, we can also conclude that our new urban area detection method is fairly fast and reliable. We can further apply probabilistic relaxation to improve our results. However, we will need extra computations to perform it. The next step in this study will be detecting the urban area type (such as dense, homogeneous, and well structured) by analyzing the voting matrix characteristics.

\section{REFERENCES}

[1] J. A. Benediktsson, M. Pesaresi, and K. Arnason, "Classification and feature extraction for remote sensing images from urban areas based on morphological transformations," IEEE Trans. Geosci. Remote Sens., vol. 41, no. 9, pp. 1940-1949, Sep. 2003.

[2] S. Bhagavathy and B. S. Manjunath, "Modeling and detection of geospatial objects using texture motifs," IEEE Trans. Geosci. Remote Sens., vol. 44, no. 12, pp. 3706-3715, Dec. 2006.

[3] L. Bruzzone and L. Carlin, "A multilevel context-based system for classification of very high spatial resolution images," IEEE Trans. Geosci. Remote Sens., vol. 44, no. 9, pp. 2587-2600, Sep. 2006.

[4] M. Fauvel, J. Chanussot, and J. A. Benediktsson, "Decision fusion for the classification of urban remote sensing images," IEEE Trans. Geosci. Remote Sens., vol. 44, no. 10, pp. 2828-2838, Oct. 2006.

[5] L. M. Fonte, S. Gautama, W. Philips, and W. Goeman, "Evaluating corner detectors for the extraction of man made structures in urban areas," in Proc. IEEE Int. Geosci. Remote Sens. Symp., 2005, pp. 237-240.

[6] V. Karathanassi, C. Iossifidis, and D. Rokos, "A texture-based classification method for classifying built areas according to their density," Int. J. Remote Sens., vol. 21, no. 9, pp. 1807-1823, Jun. 2000.

[7] V. Kyrki, J. K. Kamarainen, and H. Kalviainen, "Simple Gabor feature space for invariant object recognition," Pattern Recognit. Lett., vol. 25, no. 3, pp. 311-318, Feb. 2004.

[8] N. Otsu, "A threshold selection method from gray-level histograms," IEEE Trans. Syst., Man, Cybern., vol. SMC-9, no. 1, pp. 62-66, Jan. 1979.

[9] C. Schmid, R. Mohr, and C. Bauckhage, "Evaluation of interest point detectors," Int. J. Comput. Vis., vol. 37, no. 2, pp. 151-172, Jun. 2000.

[10] B. Sirmaçek and C. Ünsalan, "Urban area and building detection using SIFT keypoints and graph theory," IEEE Trans. Geosci. Remote Sens., vol. 47, no. 4, pp. 1156-1167, Apr. 2009.

[11] M. Sonka, V. Hlavac, and R. Boyle, Image Processing, Analysis and Machine Vision., 3rd ed. Toronto, Canada: CL Eng., 2007.

[12] C. Ünsalan, "Gradient magnitude based support regions in structural land use classification," IEEE Geosci. Remote Sens. Lett., vol. 3, no. 4, pp. 546-550, Oct. 2006.

[13] C. Ünsalan and K. L. Boyer, "Classifying land development in high resolution panchromatic satellite images using straight line statistics," IEEE Trans. Geosci. Remote Sens., vol. 42, no. 4, pp. 907-919, Apr. 2004.

[14] C. Ünsalan and K. L. Boyer, "Classifying land development in high resolution satellite imagery using hybrid structural-multispectral features," IEEE Trans. Geosci. Remote Sens., vol. 42, no. 12, pp. 2840-2850, Dec. 2004.

[15] C. Ünsalan and K. L. Boyer, "A theoretical and experimental investigation of graph theoretical measures for land development in satellite imagery," IEEE Trans. Pattern Anal. Mach. Intell., vol. 27, no. 4, pp. 575-589, Apr. 2005.

[16] M. Vetterli and J. Kovacevic, Wavelets and Subband Coding., 1st ed. Englewood Cliffs, NJ: Prentice-Hall, 1995.

[17] P. Zhong and R. Wang, "A multiple conditional random fields ensemble model for urban area detection in remote sensing optical images," IEEE Trans. Geosci. Remote Sens., vol. 45, no. 12, pp. 3978-3988, Dec. 2007. 